EOMmUn: Communication et organisation

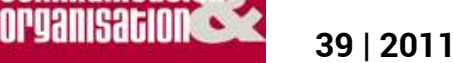

Les applications de la sémiotique à la communication des organisations

\title{
Les organisations interactives: quelle innovation pour la communication?
}

\section{Eléni Mitropoulou}

\section{OpenEdition}

\section{Journals}

Édition électronique

URL : http://journals.openedition.org/communicationorganisation/3053

DOI : 10.4000/communicationorganisation.3053

ISSN : 1775-3546

Éditeur

Presses universitaires de Bordeaux

Édition imprimée

Date de publication : 1 juin 2011

Pagination : $37-58$

ISBN : $978-2-86781-744-1$

ISSN : $1168-5549$

Référence électronique

Eléni Mitropoulou, "Les organisations interactives: quelle innovation pour la communication? », Communication et organisation [En ligne], 39 | 2011, mis en ligne le 01 juin 2014, consulté le 01 mai 2019. URL : http://journals.openedition.org/communicationorganisation/3053 ; DOI : 10.4000/ communicationorganisation.3053 


\title{
Les organisations interactives : quelle innovation pour la communication?
}

\author{
Eléni Mitropoulou'
}

Si pour étudier la communication en entreprise ${ }^{2}$ on affirmait dans les années 90 qu'il « convient de dépasser le contrôle des personnels (années 60), la gestion des personnels (années 70), ou la stratégie des ressources humaines (années 80) pour tenter de mieux comprendre la nature du lien entre l'entreprise, la société civile et l'Etat $»^{3}$, alors actuellement s'impose une sémiotique de la communication des organisations au titre de sémiotique du lien professionnel. Elle s'impose car à ce jour, à la lumière des usages des technologies numériques, nous devons focaliser sur les processus de transmission et de partage de l'information professionnelle au titre de processus producteurs de valeurs. Conformément à la position selon laquelle le sens est direction ${ }^{4}$ et, en s'appropriant la fameuse formule de Watzlawick ${ }^{5}$, en communiquant on ne peut pas ne pas signifier, nous souhaitons aborder le lien professionnel de l'organisation comme valeur promue par les technologies de l'information ${ }^{6}$ :

- Par rapport à un ordre "selon lequel des éléments sont distribués dans des rapports de co-existence ${ }^{7}$, inhérent à l'organisation comme le lieu de vie de l'information.

1. Eléni Mitropoulou est Maître de Conférences HDR à l'Université de Franche-Comté (LASELDI) - Université de Bourgogne (CIMEOS). emitropo@univ-fcomte.fr

2. Selon une conception large de celle-ci, ainsi que l'implique la notion même d'organisation : collectif à visée lucrative ou non, où les tâches sont reparties et les rôles définis en fonction d'objectifs supposés partagés - Bruno Ollivier, Les sciences de la communication : théories et acquis, Paris, Armand Colin, 2007. 3. Alain Milon, Michèle Jouve, Communication et organisation des entreprises : approche critique et cas pratiques, Rosny, Bréal, 1996, p.13.

4. Position d'abord philosophique (Henri Bergson, La pensée et le mouvant: essais et conférences, Paris, F. Alcan, 1934, p. 152), ensuite sémiotique (Jacques Fontanille, Sémiotique du discours, Limoges, Pulim, 1998, p. 21).

5. Qui précise qu'on ne peut pas ne pas communiquer, car même le refus de la communication constitue un message.

6. Appliquées à la communication interne, pilier du dispositif professionnel global.

7. Louis Marin, "Une mise en signification de l'espace social : manifestation, cortège, défilé, procession (notes sémiotiques) ", Revue Sociologie du sud-est, Sémiotique et Mass-média, n³7-38, CNRS Université de Nice, Publications Université de Provence, 1983, p. 17. 
- en fonction d'effets produits «par des opérations et des mouvements " c'est-à-direl'organisation comme un espace de promotion du lien professionnel par la circulation de l'information.

- Il s'agit de construire un projet d'explication de l'information en action car c'est par son rôle que l'organisation devient espace (ou « lieu pratiqué »") de communication au profit de la promotion du lien professionnel interne. Après une vingtaine d'années d'application des technologies de l'information en entreprise nous allons expliquer notre position et son optique selon un regard qui se situe en aval des perceptions et usages de la technologie, dans leurs effets de sens pour le lien professionnel. Aussi, en aval des expériences professionnelles du plus grand nombre ${ }^{10}$, l'univers professionnel contemporain est-il évalué comme celui de l'absence de lien de communication :

- absence de figures de direction (= il n'y a plus de patron à qui parler en l'occurrence dans les grandes ou moyennes entreprises),

- absence de rapport interpersonnel (= il n'y plus de dialogue avec ses collègues),

- absence de médiation pour l'information (= il n'y a pas de canal autre que virtuel).

Il s'agit d'évaluations qui favorisent la perception d'un manque de communication et d'une interaction dysphorique avec l'information. Pourtant, il est possible qu'il n'y ait jamais eu autant d'intention de communiquer, autant de logistique pour l'information car, de nos jours, toute (ou presque) organisation possède son site Internet, outil privilégié de la communication externe, et son site intranet, outil privilégié de la communication interne ; les deux étant essentiels à la fabrication de valeurs promues par la direction des ressources humaines.

On pourrait alors se demander s'il serait utile d'édifier une typologie de supports et de discours qui caractérise ce domaine ? Il serait peut-être utile de relever ce qui est toujours en cours d'utilisation ou ce qui est devenu obsolète - en matière de communication descendante ${ }^{11}$ par exemple comme la note de service ou le panneau d'affichage " parent pauvre de la communication interne $»^{12}$.

\section{Ibid. p. 17}

9. Michel de Certeau, Arts de faire, 1980, p. 208.

10. Ainsi que nous devons le faire a fortiori en sémiotique de la réception.

11. «Ses principales fonctions consistent à informer, à mobiliser [...], à expliquer et à fédérer le projet de l'entreprise. La communication descendante, dont la finalité principale est de résoudre les problèmes conflictuels et les tensions internes de l'entreprise, reste une communication hiérarchique, même si elle permet une meilleure cohérence au sein de l'entreprise. Son principal intérêt est d'ailleurs de véhiculer des informations réglementaires en vue d'informer les acteurs de l'entreprise de leurs tâches et de leurs fonctions ». Alain Milon, Michèle Jouve, op. cit., p 140.

12. Nicole d'Almeida, Thierry Libaert, La communication interne des entreprises, Paris, Dunod, 2007, p. 48. 
C'est sans doute utile si l'objectif est d'expliquer l'origine ou ce qui participe des évaluations dysphoriques, identifier leurs fondements et statuer sur leur pertinence.

C'est nécessaire si l'objectif est de repérer les mutations intra et inter supports de communication, tel le vidéotexte relatif à la diffusion électronique de l'information ou la lettre au personnel, désormais en ligne sur l'intranet.

C'est indispensable si l'objectif est de rendre compte du fonctionnement des dispositifs de communication en relation avec le plan de communication. En effet, étant donné la variété des supports (électroniques mais pas uniquement), les propriétés de ces supports ne peuvent pas être dissociées du plan de communication. Le choix des fonctionnalités pour la diffusion de l'information est un choix de valeur de communication, la dimension technologique n'est donc pas utilisée que pour elle-même, elle a valeur professionnelle.

Mais, il y a également le projet de la valeur sémiotique de la communication des organisations (désormais indiquée par $\mathrm{CdO}$ ) à un moment donné de sa performance où il s'agit de questionner l'Organisation comme structure d'accueil et de vie propres à l'intention de communiquer l'information. En fonction de l'actualité technologique (tels le site intranet ou la web radio d'une institution par exemple), l'organisation sur le réseau est un lieu de rencontre entre énonciations à visée interne et à visée externe ${ }^{13}$. Selon notre postulat ce lieu est un lieu de médiation où l'énonciation à visée interne transforme et se transforme par l'énonciation à visée externe.

Dans notre projet, il s'agit d'expliquer les lieux d'information comme des liens $^{14}$ de communication professionnelle car c'est l'interaction lieu/lien qui nourrit et qui détermine les postures par rapport à l'absence (ou la présence) de bonne communication, postures identifiées dans les discours tenus et partagés par le plus grand nombre, constitutifs de la doxa professionnelle et par extension sociale.

L'intranet, dispositif privilégié de la communication interne (elle-même moteur structurant de l'Organisation), devient un chef-lieu pourl'information comme actant de la relation professionnelle. C'est également pour cette raison que nous concentrons notre projet sur le lien professionnel interne de l'organisation.

\section{Organisation Interactive et savoir-faire sémiotique}

Avec la diffusion de l'information selon la technologie réseau, une organisation entre dans la sphère de la communication interactive (= par interactivité), devient une organisation du lien professionnel visiblement

13. Par définition interdépendantes; la communication interne consiste en une des déclinaisons de la communication d'entreprise - Nicole d'Almeida, Thierry Libaert, op. cit., 2007.

14. Ou des jonctions sémiotiquement parlant. 
partagéet se trouve face à une alternative : s'assumer soit en tant qu'organisation interactive soit en tant qu'organisation réactive.

Pour ce qui est de la visibilité du partage, elle se veut différente via le réseau par rapport aux processus constitutifs et fondateurs de la communication interne ${ }^{15}$. En fait, la visibilité interactive porte la valeur positive de bonne visibilité au nom d'une information performante : plus disponible, plus quantitative, plus catégorisée, plus personnalisée, plus pratique, ... toujours plus et surtout plus professionnelle.

Pour ce qui est de l'alternative organisation interactive / réactive c'est, évidemment, plus compliqué : la relation hiérarchique réactive/interactive depuis l'angle de la technologie (selon lequel la réactivité est une première étape d'interactivité) semble bien perçue lors des usages mais elle n'est pas comprise comme telle ${ }^{16}$. Mais surtout c'est bien la hiérarchie réactivité/interactivité qui fait percevoir la $\mathrm{CdO}$ soit comme une innovation pour le lien professionnel (cas de la communication interactive) soit comme une simulation de lien (cas de la communication réactive). Comment identifier dans un dispositif de la communication interne ce qui est compris comme innovation? Quelles répercussions sémiotiques pour la $\mathrm{CdO}$ comme lien professionnel?

Entre en scène l'information comme trait pertinent de cette sémiotisation : c'est en interrogeant le statut de l'information en ligne que l'on peut ériger une sémiotique comme organon conceptue ${ }^{17}$ et méthodologique au service de la $\mathrm{CdO}$, au détriment d'une sémiotique qui active les organisations pour son propre compte. Si cette dernière est, en soi, légitime, seule la première participe d'une sémiotique de la communication. Par conséquent, il s'agit d'une actualisation sémiotique pour l'organisation et dans cette perspective, il s'agit moins d'analyse sémiotique appliquée à la technologie via telle typologie d'information de telle organisation, que de l'organisation vue depuis l'information comme actant technologique.

C'est dans ce cadre que nous parlons d' innovation $^{18}$ ": l'absence de patron à qui parler ... de rapport interpersonnel ... de canal autre que virtuel ... est la manifestation d'une valeur (l'absence) érigée en problème de communication (le manque) d'un terrain professionnel (l'organisation) et solutionné par la création d'un besoin (= il faut rendre présent le lien professionnel). Cette présence est assurée par la visibilité du lien professionnel : en effet, construire

15. Note de service, lettre au personnel, panneau d'affichage, mot du président, rapport écrit, revue de presse, portes ouvertes, visite d'entreprise, journaux d'entreprise, livret informatif, dépliant, réunion, réunion d'information, réunion d'échange, compte rendu, commission, conférence, groupe de travail, cercle de qualité, l'audiovisuel, la ligne téléphonique ouverte, Alain Milon, Michèle Jouve, op. cit., pp. 140-145.

16. Tel que l'on peut le constater auprès d'enquêtes de réception menées auprès d'une centaine d'étudiants (membres de l'organisation universitaire) pour lesquels un site Internet n'est jamais qualifié de réactif mais de peu ou pas attractif ou alors de peu interactif.

17. Formule de Bruno Latour, in : Paolo Fabbri Le tournant sémiotique, Paris, Hermès Lavoisier, 2008, p. 27.

18. Jacques Fontanille, "Les métiers de la sémiotique - signification, conception, innovation », dans Métiers de la sémiotique, sous la direction de Jacques Fontanille et Guy Barrier, Limoges, Pulim, 1999. 
la nécessité de la visibilité du lien professionnel comme condition de bonne communication interne participe du processus de l'innovation.

Ce processus interpelle l'innovation porteuse de changements fonctionnel et symbolique par l'attribution d'une valeur positive ${ }^{19}$ en même temps que se construit la dégradation de l'échange professionnel. L'organisation interactive engage la valeur de visibilité de la communication professionnelle en introduisant comme problème l'échange non visible et proposant comme solution l'échange visible. Plus encore c'est l'organisation interactive qui présuppose l'univers de dégradation du lien par des plans d'expression de l'échange visible. Cette visibilité est une valeur d'innovation; nous la désignons par le terme de l'hyper-information.

\section{Un chef-lieu du lien professionnel interne}

L'exemple de l'intranet est efficace pour interroger le lien professionnel au titre d'innovation par l'information. Selon les définitions les plus répandues, l'intranet désigne l'utilisation des technologies Internet par l'accès réservé aux membres autorisés de l'entreprise (et parfois à certains partenaires). D'ancrage plus spécifiquement SIC, l'intranet est une formule de la communication électronique au titre de «banque de données interne à l'entreprise offrant à l'exemple des serveurs Internet la possibilité d'échange et de dialogue ... il faut noter que tout comme les entreprises disposent fréquemment de plusieurs journaux internes, elles gèrent aussi plusieurs intranet $»^{20}$. D'un certain point de vue, en management et en recherche d'information, par intranet «il faut comprendre le réseau interne donnant accès aux différentes applications et ressources informatiques qui y sont connectées ${ }^{21}$. Si par ailleurs « il ne s'agit pas de restreindre ce périmètre aux seuls sites web présents sur le réseau interne $»^{22}$, c'est toutefois de cela que se contentera notre étude qui, en revanche, tient bien compte du fait que « les intranet peuvent être très différents d'une organisation à l'autre en termes de contenants et de contenus $»^{23}$, car c'est ce qui nous permet de cibler les ruptures entre liens professionnels.

De ces observations, notre point de vue retient que :

- le principe d'échange et de dialogue est possible,

- l'intranet peut assumer de fonctions spécifiques,

- l'intranet est porteur de la notion d'accessibilité à l'information mais également aux dispositifs, et

- s'il n'existe pas de prototype intranet pour les organisations ${ }^{24}$, qu'en est-il au sein d'une typologie d'organisation?

19. Ibid. p. 15-26.

20. Nicole d'Almeida, Thierry Libaert, op. cit., pp. 66-67.

21. Gilles Balmisse, La recherche d'information en entreprise, Paris, Hermès Lavoisier, 2007, p. 32.

22. Ibid.

23. Ibid.

24. Ainsi que nous l'avons rapporté, les intranet peuvent être très différents d'une organisation à l'autre en termes de contenants et de contenus. 
Cette dernière question insiste sur la pertinence de la différence par rapport à l'uniformité d'un univers professionnel donné. Afin de mettre en œuvre nos réflexions et à l'épreuve nos remarques, nous convoquerons les sites Intranet des universités françaises et leur Espace Numérique de Travail (ENT). L'objectif premier de cette étude étant d'apporter un éclairage sémiotique à la $\mathrm{CdO}$, il nous faut :

1) Concrétiser la légitimité et l'intérêt de la rencontre sémiotique / $\mathrm{CdO}$ par le choix d'un exemple. Le propre de la $\mathrm{CdO}$ est de manipuler des contenus en vue de leur médiatisation, leur attribuant soit la valeur d'information interne soit la valeur d'information externe. En effet, l'interdépendance entre communications interne et externe ne doit pas confondre leurs objectifs respectifs. Pour ce qui est de ceux de la communication interne :

Beaucoup d'entreprises ont renoncé à créer de toutes pièces une "communauté de culture» qui réconcilie valeurs et objectifs de l'organisation et valeurs des individus. La tentation fusionnelle (guerrière ou tribale) est aujourd'hui largement abandonnée. La communication interne ne propose plus de faire vibrer à l'unisson. Elle n'est ni outil de propagande ni outil de manipulation. L'art de la communication interne consiste à créer un langage commun qui ne soit pas langue de bois. Contre une forte tentation communautaire il s'agit d'organiser les relations entre les individus et l'entreprise sans exclure la possibilité de différend ${ }^{25}$.

Ces observations nous interpellent à plusieurs niveaux et nous allons y revenir. Pour l'instant nous souhaitons attirer l'attention sur la question des objectifs en fonction de la distinction entre entreprise et individus, distinction qui marque une rupture avec la conception selon laquelle l'entreprise, ce sont les individus. À priori, la communication interne se voudrait le retour à la communication du lien hiérarchisé par abandon de la vision idéaliste d'un actant collectif paradigmatique. L'information interne participe pleinement de ce cadre relationnel : sa conception, sa diffusion, sa réception sont indissociables de ce qui " engage une globalisation de l'action, une coordination des messages, des moyens et des publics qui réconcilient les différentes facettes de l'entreprise ${ }^{26}$, que cette entreprise soit une structure lucrative, une institution, une administration, une association.... c'est-à-dire une structure caractérisée par des procédures de mise en relation par l'information. L'université, en l'occurrence française, relève d'une telle structure. La sémiotique étant en mesure d'expliquer la structuration de l'information dans un processus de sa communication comme processus de culture ${ }^{27}$, ici, professionnelle, elle s'avère compétente pour interroger le contrat de communication de l'université.

2) Réfléchir à l'ENT en fonction d'une sémiotique qui tend vers la $\mathrm{CdO}$ et non le contraire. Il est primordial de faire intervenir la pertinence de la sémiotique comme point de vue non dogmatique mais spécifique sur la

25. Nicole d'Almeida, Thierry Libaert, op. cit., p. 30, souligné par nous.

26. Nicole d'Almeida, Thierry Libaert, op. cit., p. 9-10.

27. Umberto Eco, La structure absente, Introduction à la recherche sémiotique, Paris, Mercure de France, 1972. 
$\mathrm{CdO}$, actualisée comme domaine des pratiques professionnelles de l'Homme (universitaire) en fonction des outils qu'il développe dans ce cadre et des relations que cela génère avec les autres formes de communication du milieu de travail (sans oublier qu'elles sont désormais difficilement dissociables de celles de la vie privée).

3) Sonder la fonction de l'information interne afin d'identifier la valeur des procédures de sa manipulation ${ }^{28}$ et rendre compte de ses répercussions sémiotiques sur le lien professionnel. Dans quelle mesure le dispositif ENT est-il une innovation pour le lien professionnel intra-université ? Permet-il de franchir le seuil du support pour celui du medium de la communication professionnelle ? Car le passage de support à medium marquerait l'apport d'une valeur ajoutée en communication ${ }^{29}$.

Aussi, l'intérêt de l'éclairage sémiotique se situe au niveau de la nébuleuse de la forte transversalité des supports et au coeur du rapport organisations / information / numérique.

La spécificité primaire de la $\mathrm{CdO}$ étant une forte intentionnalité (une organisation a fortiori ne peut pas ne pas communiquer), le devoir communiquer lui est substantiel. Une organisation procède donc par stratégie de décision sémiotique $^{30}$ de la médiatisation de l'information. Il s'agit de décider non seulement quelle information sera diffusée et à qui (c'est là une évidence) mais surtout de décider selon quelles fonctions sémiotiques et cela relativement au processus de médiatisation de l'information. Ainsi, une université qui utilise un site Internet (ce qui est, a priori, commun aux universités françaises) peut le faire :

- Par complémentarité informationnelle (les mêmes informations mises en ligne et hors ligne ${ }^{31}$ ) et exclusivité communicationnelle de l'information (les informations participent de contrats de communication hétérogènes ${ }^{32}$ ).

- Par complémentarité communicationnelle liée au support hors ligne (flyer, vidéo, affiche ... qui créent une polyphonie médiatique) et exclusivité informationnelle (autres informations en ligne que dans le flyer, par exemple).

- Par création de relations inédites entre supports matériel/formel ${ }^{33}$, et à ce titre l'on peut se demander si l'ENT prolonge ou innove la valeur communicationnelle de l'information interne.

28. Manipulation au sens sémiotique (c'est-à-dire ni positif ni négatif) contrairement au sens communicationnel tel que l'implique la définition précédemment citée par outil de manipulation.

29. Eléni Mitropoulou, "Vers une sémiotique du medium : une sémiotique à légitimer ? ", Nouveaux Actes Sémiotiques, 2008, http://revues.unilim.fr/nas/.

30. Jean Marie Klinkenberg, Précis de sémiotique générale, Bruxelles, De Boeck, 1996, p. 64-65.

31. Tels les magazines d'information (supports papier et virtuell) « Tout l'U » et « En Direct » de l'université de Franche-Comté.

32. Du point de vue du contrat de communication, magazines papier et en ligne n'ont rien à voir pour des raisons autant matérielles que formelles.

33. Jacques Fontanille, "Du support matériel au support formel ", dans Marc Arabyan, Isabelle Klock-Fontanille (éd.), L'écriture entre support et surface, Paris, L'Harmattan, 2005, p. 183-200. 
L'on peut également se demander si l'ENT est gage de modernité ou bien trait pertinent de communication ? Si l'ENT participe d'une obligation de communication en interne (un devoir faire) ou d'une envie de communiquer en interne (un vouloir faire). L'on peut enfin se demander si l'interactivité et la multimodalité, caractéristiques de la carte d'identité médiatique (soit la charte graphique) de l'ENT assument une valeur référentielle (favoriser l'interaction entre membres de l'université) ou mythique (favoriser l'image d'une université en interaction). Puisque l'ENT fonctionne comme vitrine (c'est une communication visuelle qui met en valeur des valeurs), il s'agit de poser la question de l'ENT en termes d'outil au service de la relation professionnelle entre les composantes de l'université. Composante implique les instances in presentia (salariés, direction, étudiants) et in absentia (partenaires et public externe). En effet, une université est un syncrétisme d'acteurs, ce n'est pas simplement une instance émettrice d'information selon un mouvement seulement excentrique mais une instance à mouvement également concentrique, telle la conception de Kandinsky pour les couleurs chaudes/froides. De la même façon que le mouvement d'une couleur inclut sa réception ${ }^{34}$, une université qui communique par l'ENT le fait selon ce double mouvement fondamentalement concentrique (puisque tourné vers l'intérieur ${ }^{35}$ ), toutefois parfois excentrique (tourné vers l'extérieur), plus rarement les deux. Arrêtons nous sur une série d'exemples et commentons-les en fonction de notre remarque : prenons une université qui communique par $E N T$, car contrairement à ce que l'on pourrait penser, toutes les organisations universitaires publiques n'ont pas de page ENT visible ${ }^{36}$ telles que les universités de Savoie, de Grenoble Pierre Mendès France ou de la Haute Alsace. Pas d'ENT visible n'implique pas absence de communication interne en ligne mais absence d'espace informationnel visiblement dédié à cet effet. Pour l'université de Savoie c'est par le site Internet que nous pouvons accéder à la communication interne ${ }^{37}$ moyennant les codes nécessaires (login + mot de passe) situés en haut à gauche :

34. Comme par exemple le bleu qui « est animé d'un mouvement concentrique que l'on peut comparer à celui de l'escargot qui se rétracte dans sa coquille. II s'éloigne de l'observateur », Kandinsky, Du spirituel dans l'art et dans la peinture en particulier, Paris, Denoël Médiations, 1969, p. 119-120.

35. Le fait que de nombreux ENT ne sont accessibles qu'avec code d'accès (nous en parlons un peu plus loin) réservé et attribué aux personnels de l'université détermine l'ENT comme outil de la communication interne. 36. à la date de cette étude, exemples consultés les 8 et 21 juin 2011.

37. Contrairement à celles de Mulhouse ou de Bourgogne, nous en parlons plus loin. 


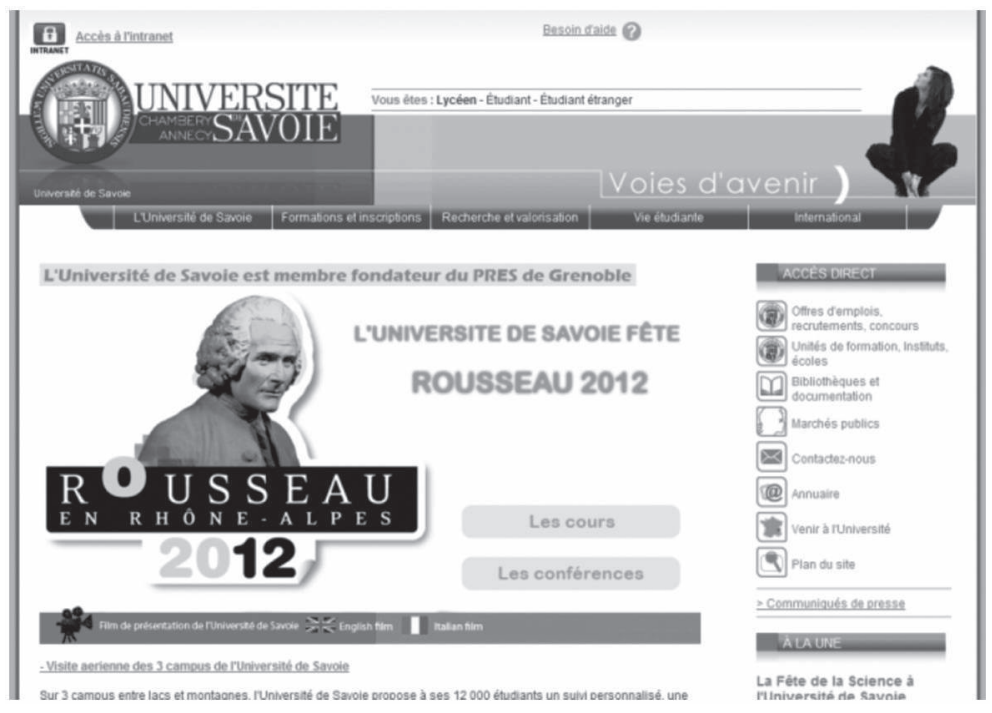

Là, l'externe est un palier pour l'interne, l'interne niche dans l'externe, le mouvement de l'information excentrique absorbe celui de l'information concentrique, comme dans l'exemple des Antilles-Guyane où l'intranet manque de visibilité, le lien vers elle étant situé tout en bas d'une longue page d'accueil :

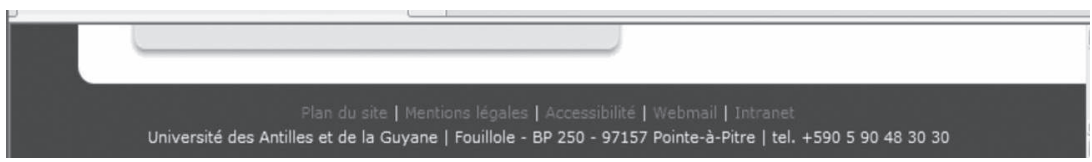

suivi d'une demande de code d'accès :

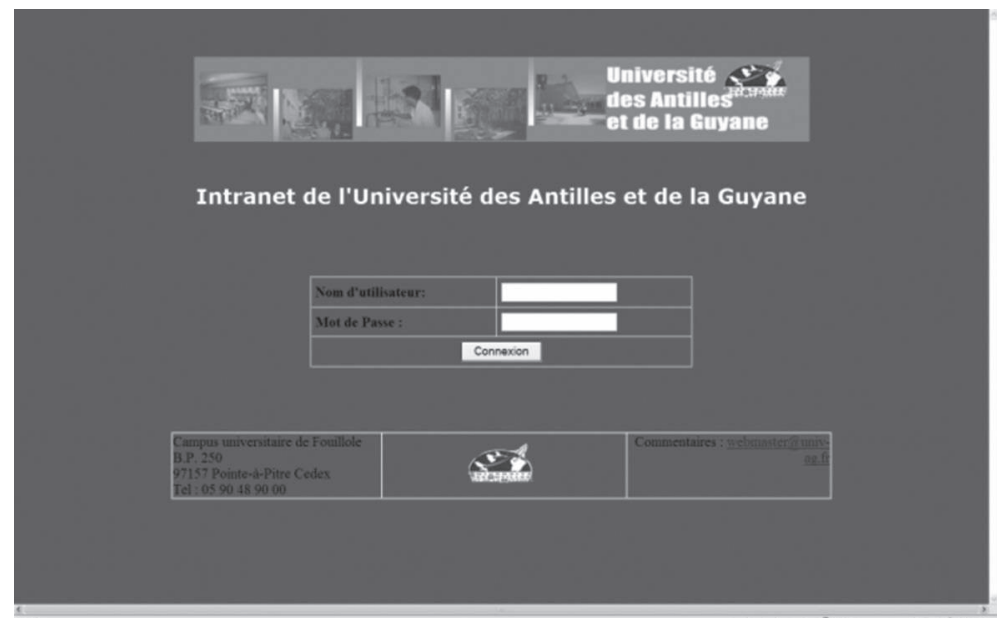


contrairement à la majorité des sites Internet universitaires qui proposent un accès non réglementé à la page ENT tel celui de Franche-Comté (UFC), dont le lien est situé en bas à droite, soit dans la scène 4 assumant la fonction de sous-scène ${ }^{38}$ dédiée à la communication de l'entreprise à proprement parler ${ }^{39}$ :

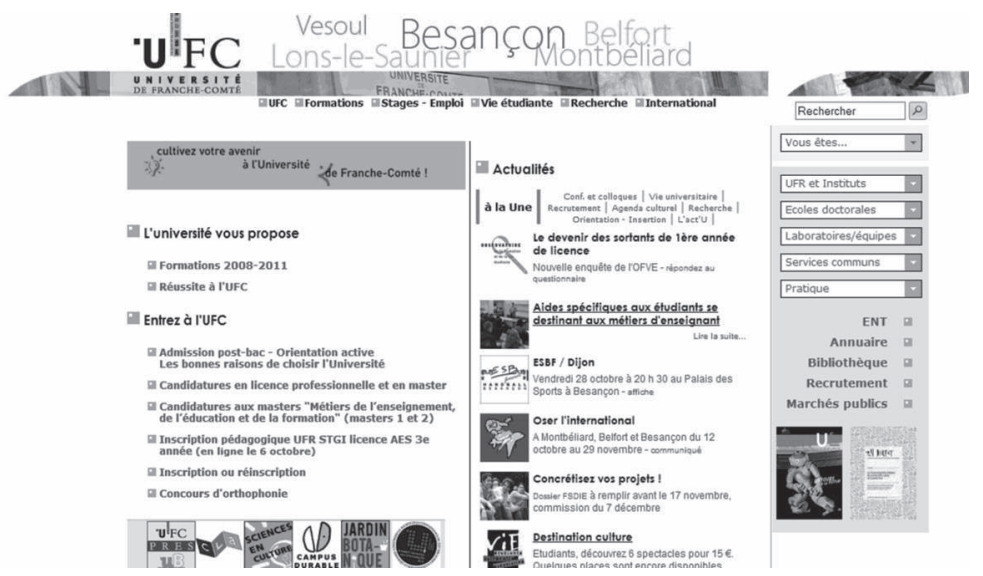

Si l'externe sert aussi de palier à l'interne, celui-ci se donne à voir dans l'externe, et une fois le lien ENT activé il donne à voir, sans demande de code d'accès, le lieu pratiqué de l'information :

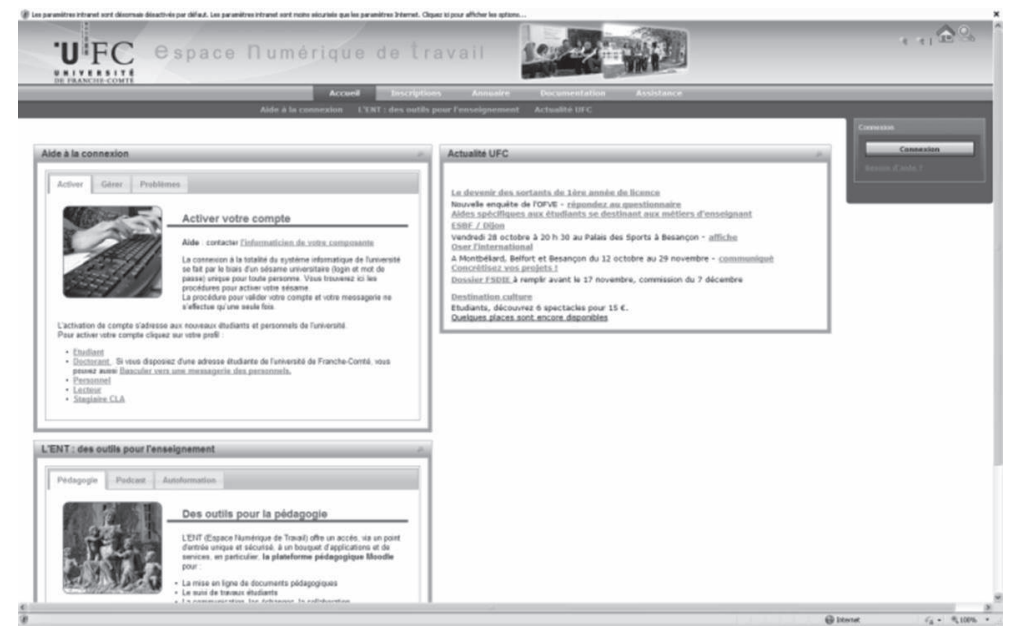

avant d'exiger dans un troisième temps seulement, un code d'accès :

38. Selon la description initiée par Peter Stockinger, 2003, www.semionet.fr.

39. Par rapport aux communications produit, marque et institutionnelle. 


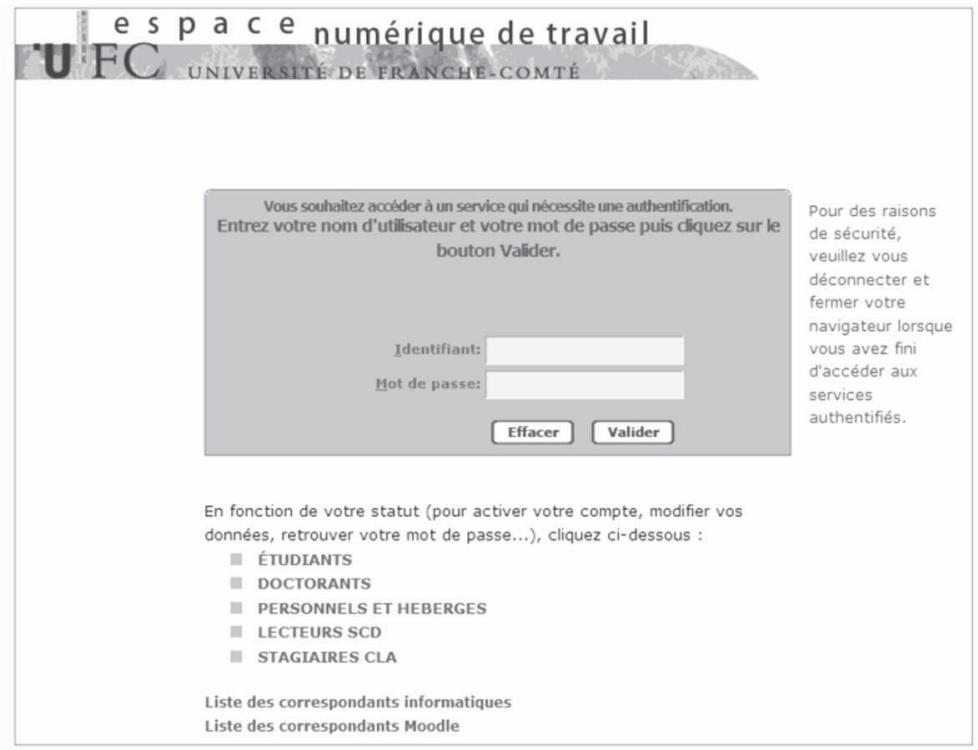

Contrairement aux exemples de Savoie et d'Antilles-Guyane où le site Internet est le seul espace de communication visible de l'extérieur, nous constatons la volonté de montrer l'intérieur organisationnel, ce qui participe d'une certaine logique organisationnelle évidente : l'ENT en tant qu'espace de communication interne n'existe qu'en interne, c'est donc uniquement son existence qui se rend visible de l'extérieur. De façon peut-être plus radicale l'université de Grenoble 1 ne propose pas d'ENT, ce qui est également le cas de l'université de Haute-Alsace. Il s'agit là d'une absence totale de visibilité pour la communication interne, ce qui, de nouveau, ne veut pas dire qu'elle n'existe pas, mais que, si elle existe, elle ne se donne pas à voir à l'extérieur.

Cette nuance en dit long sur une sorte de signification paradoxale : malgré le principe concentrique fondateur de la communication interne ${ }^{40}$, l'absence de visibilité pour l'ENT implique lors de la réception en ligne l'absence de dispositif de communication interne. Plus encore : pourquoi ce qui est par définition non visible de l'extérieur (le processus de communication interne) est perçu comme une carence de communication ? Cette signification paradoxale n'est-t-elle pas celle d'une pratique confisquée par l'absence affichée de volonté d'échanger?

En revanche, mais au profit d'une autre sémiose paradoxale, la présence de visibilité du plan d'expression ENT via le site Internet (tel le cas de l'UFC) construit la présence de volonté de communication interne : ce que tout le monde peut voir n'est plus de l'ordre de l'interne... Qu'en est-il de la communication interne comme outil de propagande ou outil de " manipulation » ${ }^{41}$ ? Un autre

40. Ce qui ne doit pas être confondu avec l'articulation incontournable entre interne et externe. 41. Ibid. 
cas est à signaler, par exemple celui de l'université de Bourgogne qui affiche une ENT personnalisée (cf. L'ENT : mon espace numérique) par accès direct depuis Google :

Google université de bourgogne Recherche avancée

Recherche Environ 639000 résultats $(0,13$ secondes $)$

\begin{tabular}{|c|c|c|}
\hline Tout & \multicolumn{2}{|c|}{$\begin{array}{l}\text { Université de Bourgogne } \\
\text { www.u-bourgogne.ff/ - Bloquer tous les résultats de www.u-bourgogne.ff } \\
\text { il y a } 1 \text { jour - Présentation de l'université de Bourgogne, les formations, la recherche, } \\
\text { la vie à l' uB, les services en ligne, les rubriques étudiants et futurs ... }\end{array}$} \\
\hline Vidéos & $\frac{\text { L'ENT: mon espace numérique }}{\text { www.u-bourgogne. ff/-Mon-espace-... }}$ & $\frac{\text { Comment m'inscrire }}{\text { www.u-bourgogne.ff/-Comment-m-i... }}$ \\
\hline Actualités & L'Université met à votre ... & Vous souhaitez vous inscrire à ... \\
\hline Shopping & $\begin{array}{l}\text { Les formations } \\
\text { www.u-bourgogne ff/-Les-formatio... } \\
\text { Université de Bouraogne }\end{array}$ & $\frac{\text { L'UB recrute }}{\text { www.u-bourgogne.ff/L-LB-recrute-... }}$ \\
\hline rids & UFR, Instituts, Ecoles & Bibliothèque de l'université de ... \\
\hline $\begin{array}{l}\text { Besançon } \\
\text { Changer le lieu }\end{array}$ & $\begin{array}{l}\text { Www. u-bourgogne ffr-UFR-Instituts-... } \\
\text { Consultez la liste des facultés ... }\end{array}$ & $\begin{array}{l}\text { scd.u-bourgogne.ff/ } \\
\text { Catalogues des différentes ... }\end{array}$ \\
\hline $\begin{array}{l}\text { Le Web } \\
\text { Pages en français } \\
\text { Pays : France }\end{array}$ & $\begin{array}{l}\text { BP 27877, } 21078 \text { Dijon } \\
0380395000 \\
2 \text { avis de Google - Itinéraire - Est-ce corre }\end{array}$ & \\
\hline
\end{tabular}

qui ouvre la page ENT sans demande de code préalable :

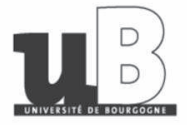

UNIVERSITÉ

Dolitiqus- Organisation- PRES- Las
campus de lue - UeR, Instituss, Ecoles.
Campus innovant- Développement

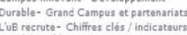

L'ue recrute- Chil
Marchés publics

LES FORMATIONS

A-rmation intitiale- Formation doctorate-

Apprentissage - Formation continua at

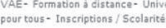

RECHERCHE ET VALORISATION

Unitas de recherche. Hablitatian

Valoristion at transfert de technologita

recherche pour tous - Publications
reser

INTERNATIONAL

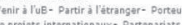

Je projets internationaux - Partenariats

Programms - Equipe / Contact

INSERTION PROFESSIONNELLE

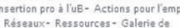

Pinsertion pro

VIE SUR LES CAMPUS

Giblichthaues - Logement/ RestavrationHandicap- Bourses / aides socialss-

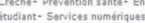
(n...n-
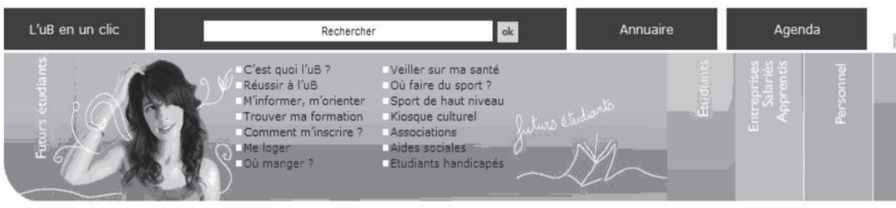

L'ENT : Mon espace numérique

L'Université met à votre disposition de nombreux services numériques (TIC). L'Espace Numérique de Travail (ENT) est le point d'entrée unique pour accéder à ces services.

L'ENT vous permet, à partir d'un navigateur Internet d'accéder à un bouquet de services et des informations personnelles, depuis n'importe quel lieu et à n importe quel moment.

Accessible à http://ent.u-bourgogne.fr, cet espace de travail vous propose :

$\triangle$ Accès à votre messagerie électronique :

Dès votre inscription à lUniversité vous disposez d'une adresse de courrier électronique prenom_nom @etu.u-bourgogne.f:

Les avantages de la messagerie étudiante

- Faciliter la communication avec l'Université : différents services de l'uB pourront vous contacter par courrie

- Valoriser vos communications avec \& l'extérieur $>$ : vous bénéficiez d'une adresse \& professionnelle * qui joue le rôle d'une carte de visite sérieuse

$\triangle$ Gérer vos signets:

Ajouter, gérer vos liens favoris (signets) sur le web pour les retrouver sur n'importe quel poste.

$\triangle$ Un onglet Scolarite

administrati, vos informations personnelles

- téléchargez et imprimez votre certificat de scolarité

- consultez le calendrier et les résultats de vos examens

$\triangle$ Un onglet Documentation:

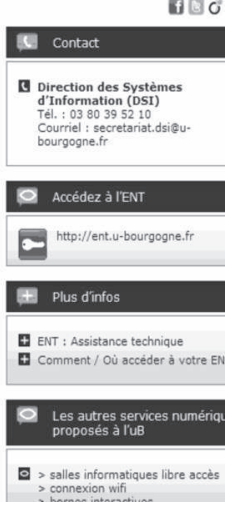


ni passage obligé par le site Internet et pour cause : le site Internet ne propose pas de lien ENT. Ici, le lien interne / externe se construit selon une interdépendance hiérarchisée où interne et externe occupent une place déterminée dans la communication d'entreprise : nous retrouvons le principe fondateur de la relation interne et externe.

Ces exemples, par la configuration du lien hypertexte comme lien professionnel, mettent en place une répartition de l'information professionnelle par un choix réactif : il s'agit pour l'usager d'occuper une place avant de pouvoir aller plus loin.

Enfin, il y a également ces cas que l'on pourrait désigner de codification ostentatoire, propres à quelques universités comme celles de Caen, de Corse ou de Jussieu, qui exigent un accès par code, interposé entre site Internet et page ENT :

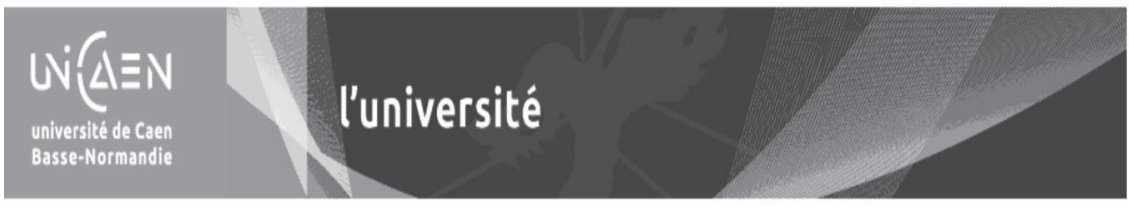

\section{Service d'authentification centralisée}

Vous souhaitez accéder à un service protégé proposé par luniversité de Caen Basse-Normandie.

Étudiants

Entrez le nom d'utilisateur et le mot de passe de votre Etup@ss.

Personnels et autres utilisateurs

Entrez le nom dututilisateur et le mot de passe de votre Persop@ess.

Besoin d'aide?

Espace numérique étudiant

Espace numérique des personnels

Pour des raisons de sécurité, veuillez vous déconnecter et fermer votre navigateur lorsque vous avez fini d'accéder aux services authentifies.

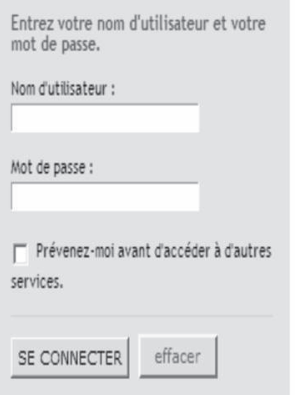

Ces cas produisent une information à usage exclusivement interne avec la création d'une frontière entre interne et externe au profit de la perception d'une organisation du lien interne préservé (exemple de Jussieu) voire cultivé (exemple de Bourgogne).

Qu'en est-il de la communication interne comme art de créer un langage commun qui ne soit pas "langue de bois ${ }^{42}$ ? Cette frontière interne/externe 
à voir en interne et en externe porte une dimension du politiquement correct en matière de communication :

- Dans le cas du lien préservé une structure favorisant le lien professionnel entre ses membres par la création d'un univers clos (information interne concentrique) ; qu'en est-il de la communication interne qui «ne propose plus de faire vibrer à l'unisson $»^{43}$ ?

- Dans le cas du lien cultivé une structure qui favorise le lien professionnel entre ses membres par la médiatisation même de ce lien (information interne excentrique). Qu'en est-il de ces entreprises qui n'ont peut-être pas renoncé à la tentation fusionnelle ${ }^{44}$ ?

En fait, l'ENT est l'outil de la communication interne en tant que visible / non visible par l'externe et l'interne.

À partir de la hiérarchisation ${ }^{45}$ entre information pour les membres de l'organisation et information pour le public hors organisation ${ }^{46}$, le couple Internet / intranet construit le lien professionnel par la manipulation de l'accès à l'information selon des éclatements variables: information attribuée, appropriée, détournée, simulée ... en tout cas information éclatée ${ }^{47}$. Tel le principe de vue (ou perspective) éclatée permettant d'identifier les pièces constitutives d'une construction, l'information universitaire se configure variablement dans les pages d'écran de la communication interactive. Toutefois, malgré cette variabilité, l'information interactive se caractérise par des éclatements autres que l'information hors ligne ${ }^{48}$.

\section{Le lien professionnel de l'Organisation interactive}

Postulant qu'il n'est pas adéquat d'isoler l'organisation interactive de la fonction de l'information, et suite à l'étude préalable de nombreux sites ENT, nous proposons de réunir les propriétés de l'information en ligne. Selon la réflexion sémiotique sur la $\mathrm{CdO}$, les données du tableau suivant ciblent le rôle de l'information dans la création du sens pour le lien professionnel. La première colonne concerne l'information en ligne de façon générique ; en revanche, la seconde colonne (divisée en deux) focalise sur les répercussions sémiotiques de l'information sur le lien professionnel (voir tableau 1).

43. Ibid.

44. Ibid.

45. Il va de soi que ce n'est pas sur le principe mais sur les modalités de la hiérarchisation qu'il s'agit d'attirer l'attention ici.

46. Car l'accès non codifié aux horaires de la BU par exemple est par excellence une information à valeur interne pour les ayant droit.

47. Jacques Fontanille, Le savoir partagé : sémiotique et théorie de la connaissance chez Marcel Proust, Paris, Hadès-Benjamin, 1987.

48. Nous ne tenons pas compte de l'accès à l'information en fonction du support numérique matériel, ce dernier introduisant à son tour d'autres variables qui n'ont pas valeur de pertinence ici car notre projet porte sur les traits invariants de l'information. 
Ces remarques sur le lien professionnel par byper-information ${ }^{49}$ (désormais HI) ne sont pas spécifiques de la communication interne des organisations : il s'agit là de l'information en ligne dans ses fonctions génériques, diffusée autant en interne qu'en externe. Toutefois, ces spécificités génériques sont nécessaires pour expliquer comment la HI influence (par principe d'innovation gestionnaire ${ }^{50}$ ) la communication interne. Si toute sorte de texte peut avoir les propriétés fonctionnelles de la $\mathrm{HI}$ (la convocation à une réunion, la vidéo d'une conférence, un bilan financier, un compte-rendu du CA, ...), ces textes, propres à l'organisation, se trouvent dotés de la même valeur de communication. Cette valeur se caractérise par :

- L'accès réservé pour tous en fonction du profil professionnel de l'HI et non de l'usager : le code attribué à tous les usagers (login et mot de passe) affiche des seuils de rétention d'information variables pour la communication ${ }^{51}$ (d'abord entre interne et externe et ensuite en interne).

- La dimension cosmopolite de l'HI. Accueillante et accommodante par sa valeur fonctionnelle ${ }^{52}$, il faut toutefois faire le pas vers l'HI : malgré les méandres du butinage, on n'arrive pas par hasard sur un site Internet universitaire. Une fois en face de l'organisation interactive, les spécificités techniques (interactivité, multimodalité) et symboliques (disponibilité, partage, attractivité, volume) de l'HI étayent les valeurs professionnelles par le type d'interaction entre interne et externe c'est-à-dire le type "d'influence réciproque qui s'opère inévitablement » entre interne et externe " mises en présence $»^{53}$ par l'interactivité.

- Si l'interactivité est une actualisation (principe de disjonction par éclatement de l'information) tandis que l'interaction serait réalisation (la transformation opérée par l'information) ${ }^{54}$, l'HI du lien interne / externe se distingue :

- de l'information du lien professionnel hors ligne par des fonctionnalités permettant de gérer la visibilité interactive (optimisation du lien professionnel) par opposition à la visibilité réactive (ranimation du lien professionnel) ${ }^{55}$,

49. Nous retenons de cette notion, importante en intelligence territoriale et en veille technologique, la propriété technologiquement performante de l'information électronique.

50. Christelle Fourrier, Fabienne Martin-Juchat, Valérie Lépine, "Y-a-t-il un modèle de communication dominant dans les organisations ou une hybridation dans les pratiques des communicants? Retour réflexif du terrain », Actes du 17ème colloque de la SFIC, Au coeur et aux lisières des SIC, Dijon, 2010, p. 437- 443.

51. même si l'accès aux types d'information et les listes de diffusion étant élaborés, évidemment, en fonction de catégories de personnel.

52. Contrairement à l'information de la presse écrite qui peut l'être par valeur éditoriale.

53. Gilles Amado, Andrée Guittet, Dynamique des communications dans les groupes, Paris, Armand Colin, 2009, p. 42.

54. Eléni Mitropoulou, Média, multimédia et interactivité : jeux de rôles et enjeux sémiotiques, Nouveaux Actes Sémiotiques, 2007, http://revues.unilim.fr/nas/

55. Ibid. 


\begin{tabular}{|c|c|c|}
\hline \multirow{2}{*}{$\begin{array}{c}\begin{array}{c}\text { Linformation comme dispo- } \\
\text { sitif technique }\end{array} \\
\text { Linfo factuelle (fonctionnelle) }\end{array}$} & \multicolumn{2}{|c|}{$\begin{array}{l}\text { Linformation comme dispositif symbolique } \\
\text { issu du technique }\end{array}$} \\
\hline & $\begin{array}{l}\text { L'info factuelle signifiée comme } \\
\text { atout (ou adjuvant) pour le lien } \\
\text { professionnel }\end{array}$ & $\begin{array}{l}\text { L'info factuelle signifiée comme } \\
\text { contrainte (ou opposant) pour le lien } \\
\text { professionnel }\end{array}$ \\
\hline $\begin{array}{l}\text { Diffusée par liste profession- } \\
\text { nelle }^{56} \text {. } \\
\text { Principe Un vers Tous. }\end{array}$ & $\begin{array}{l}\text { Information visiblement } \\
\text { partagée }^{57} \text {. }\end{array}$ & $\begin{array}{l}\text { Pas d'aller/retour. } \\
\text { Communication unidirectionnelle } \\
\text { fonctionnelle. }\end{array}$ \\
\hline Téléchargée & $\begin{array}{l}\text { Information appropriée } \\
\text { (dimension réfléchie de son } \\
\text { acquisition). }\end{array}$ & $\begin{array}{l}\text { Information attribuée } \\
\text { (dimension transitive de son acquisition). }\end{array}$ \\
\hline À temps réel & $\begin{array}{l}\text { Information mise à jour } \\
\text { Immédiate et actualisée. }\end{array}$ & $\begin{array}{l}\text { Surcharge d'information. } \\
\text { Hyperactivité du lien professionnel } \\
\text { (pathologie de l'information). }\end{array}$ \\
\hline Codifiée & $\begin{array}{l}\text { Information personnalisée, } \\
\text { sécurisée. }\end{array}$ & Information soumise à la technologie. \\
\hline Rétroactive & $\begin{array}{l}\text { Frontières de la communication } \\
\text { descendante/ascendante revisi- } \\
\text { tées grâce à l'information. }\end{array}$ & $\begin{array}{l}\text { Communication ascendante diluée à } \\
\text { cause de l'information. }\end{array}$ \\
\hline Fonctionnalisée & Information efficiente. & $\begin{array}{l}\text { Information qui exige des compétences } \\
\text { pour y accéder }\end{array}$ \\
\hline Mutualisée & $\begin{array}{l}\text { L'information porte l'identité } \\
\text { collective. }\end{array}$ & $\begin{array}{l}\text { Linformation produit des effets de } \\
\text { massification. }\end{array}$ \\
\hline Récursive & $\begin{array}{l}\text { Information accessible en } \\
\text { permanence. }\end{array}$ & Trop d'information tue l'information. \\
\hline Enchâssée & Information dilatée, augmentée. & Information dispersée. \\
\hline Localisée $\mathrm{S}^{59}$ & $\begin{array}{l}\text { Information obtenue par accès } \\
\text { privilégié. }\end{array}$ & Information sous contrôle. \\
\hline Délocalisée $e^{60}$ & $\begin{array}{l}\text { Information nomadisée (autre- } \\
\text { ment accessible). }\end{array}$ & $\begin{array}{l}\text { Information médiatée (besoin de } \\
\text { support). }\end{array}$ \\
\hline De proximitét & Information disponible. & Information encombrante. \\
\hline $\begin{array}{l}\text { Création d'un cadre de } \\
\text { communication interactive par } \\
\text { l'Information }\end{array}$ & $\begin{array}{l}\text { Inscription de l'observateur } \\
\text { comme informateur }^{62} \text { dans le } \\
\text { plan de communication interne } \\
\text { et inversement. }\end{array}$ & $\begin{array}{l}\text { Inscription variable }{ }^{63} \text { de l'observateur } \\
\text { comme Informateur dans le plan de } \\
\text { communication interne et inversement. }\end{array}$ \\
\hline
\end{tabular}

\section{Tableau 1 : Le lien professionnel de l'Organisation interactive}

56. Ainsi que nous l'avons souligné selon l'opposition listes de diffusion / discussion, Eléni Mitropoulou, "De la pratique médiatique comme topographie d'une mémoire collective», dans Nicole Pignier et Michel Lavigne (éd.), Revue MEI, Paris, L'Harmattan, p. 87-97.

57. Telle la diffusion d'une note de service dans la boîte aux lettres de tous mais qui ne veut pas dire partagée.

58. Algirdas Greimas, Joseph Courtès, Dictionnaire raisonné de la théorie du langage, Hachette Université,

1979, p. 2-3.

59. Institutionnellement

60. Accessible hors lieu de travail soit linformation du point de vue de ses fonctionnalités.

61. Accessible par le réseau soit l'information du point de vue de ses usage

62. Jacques Fontanille, op. cit., 1987.

63. Selon spécificités dispositif ENT 
de l'HI non spécifique aux organisations par la relation sociale qu'elle introduit dans le lien professionnel. Si l'information " n'est pas un objet mais une relation posée face à l'objet " ${ }^{64}$ et "une relation qui s'établit entre un objet et un regard $~^{65}$, alors l'ENT construit le regard, regard censé percevoir le passage de la communication verticale à la communication transversale mais par illusion de communication horizontale. L'organisation interactive (soit le lien professionnel par l'HI) intervient au cœur de la relation entre communications descendante / ascendante au titre de communication mutuell ${ }^{66}$ et revendique l'innovation.

\section{Information et Innovation : conclusions et perspectives}

Si nous confrontons ces modes d'intervention aux 5 critères sémiotiques de l'innovation ${ }^{67}$ :

1 - Lorganisation interactive expose l'université (soit un univers professionnel perçu clos) en la signifiant comme une structure du fort lien professionnel interne; que ce lien dissocie la communication interne de la communication externe ou que ce lien articule la communication interne à la communication externe, il est visible.

2 - L'accès, toujours calculé, est construit pour être perçu et implique l'usager comme actant.

3 - L'organisation interactive ajoute et déplace la valeur de communication par l'HI comme catalyseur biérarchique, l'IH ainsi qu'il a été souligné précédemment, déclenche et construit des seuils de réception.

4 - La CdO surgit, à la fois, comme un problème d'information et sa solution (= l'HI).

5-L'HI actualise le lien professionnel au nom de la performance technologique du contrat de communication.

La communication interactive vise les pathologies informationnelles de la $\mathrm{CdO}$ érigeant l'information hors ligne en problème (de quantité, de diffusion, de réception, de partage) et proposant l'HI comme solution. Il s'agit de la diffusion quantitative d'une réception partagée (cf. nos remarques dans la dernière ligne du tableau).

L'ENT éclate l'information, installe une scène distribuant le rôle d'observateur $^{68}$ à l'étudiant, à l'enseignant-chercheur, au chercheur, à

64. Yves Jeanneret, Y-a-t-il (vraiment) des technologies de l'information ? Villeneuve d'Ascq, Presses universitaires du Septentrion, 2011, p. 112.

65. Ibid, p. 87, nous soulignons.

66. «Elle a pour objectif d'unir les différents intervenants de l'entreprise», Alian Milon, Michèle Jouve, op. cit., p. 149

67. 1. Elle modifie notre représentation sémantique d'un domaine d'activité - 2. Elle est perceptible, orientée, et implique l'observateur - 3. Elle ajoute et déplace de la valeur - 4. Elle infléchit les attentes -5 . Elle réactualise et revalorise la promesse et le contrat avec l'usager. Jacques Fontanille, 1999, op. cit., p. 20.

68. Jacques Fontanille, 1987, op. cit., p. 74. 
l'administrateur... au titre d'informateur: ils savent qu'il y a quelque chose à partager et à faire-partager, l'HI.

Aussi, par écho aux modèles de réseau de communication ${ }^{69}$, l'HI s'interposerait-elle pour configurer un réseau étoile en réseau tous canaux, c'est-à-dire introduire la rupture avec un réseau centralisé au profit d'un réseau non centralisé. Toutefois, du fait que « la centralisation accroît l'efficacité du groupe et définit un rôle organisateur $»^{70}, l^{\prime} \mathrm{ENT}$ investit (malgré tout) la place centrale du modèle étoile (assurant la transmission rapide de l'information mais également la génération de surcharge d'information). Ce statut composite pour l'ENT (caractéristique de la circulation par HI) lui confère une fonction de transversalité, laquelle du fait qu'elle implique le service de la direction, peut faire assimiler transversalité des échanges à horizontalité des échanges ${ }^{71}$.

Le dispositif web radio $^{72}$ constitue un autre exemple pertinent de la communication par l'HI. Explicitement située sur l'articulation interne / externe ${ }^{73}$ mais destinée prioritairement au personnel de l'université, la web radio est le lieu pratiqué de la réalisation du lien professionnel à trois temps : de la communication interne en communication interne événementielle par la promotion du lien professionnel, promotion tournée vers l'extérieur par accès libre à la mise en ligne de conférences, du journal d'actualités universitaires, de cérémonies scientifiques, du journal culturel de la ville... Cette dernière intégration de l'extérieur de l'université (la ville) à l'intérieur de l'université participe de la configuration de la communication institutionnelle en communication communautaire propre au rôle social de l'organisation. La web radio étant accessible sans code via le site Internet, les frontières entre interne et externe sont organisées au profit de leur effacement. La web radio incarne le lien universitaire macro-communicationnel : l'université dans la ville et dans les réseaux sociaux (Facebook), l'université dans l'université (principe de la boîte à idées)... L'événement scientifique est au service d'une cohérence professionnelle comme cohésion sociale par l'effet récursif de l'HI : l'outil Facebook dans l'outil web radio, dans l'outil

69. Gilles Amado, Andrée Guittet, op. cit., p. 61 et suivantes.

70. Ibid., p. 62.

71. La messagerie électronique par liste de diffusion des catégories professionnelles est un exemple de cette horizontalité simulée.

72. Afin de rester dans le même cadre organisationnel citons "Résonances » de l'université de Limoges, accessible depuis le site Internet de l'organisation universitaire homonyme.

73. «Espace d'expression et de communication de tous les étudiants, enseignants et personnels administratifs de l'Université de Limoges, Résonances propose des interviews, des émissions, des reportages, des cours et des conférences. Son premier objectif est de susciter et d'améliorer la communication interne entre les composantes de l'université, à tous les niveaux souhaitables : organisation administrative, services disponibles, cursus de formation proposés, choix pédagogiques, axes et projets de recherche, débouchés envisagés, partenariats, etc. De façon à préciser les représentations et connaissances des uns et des autres, à alimenter le sentiment d'appartenance à une unité universitaire commune et cohérente. Le second objectif vise l'environnement externe de l'Université, le grand public, pour contribuer à construire une représentation juste et méliorative d'une université proche et familière ", www.unilim.fr, consulté le 7 juin 2011. 
site intranet, dans l'outil site Internet. Le principe d'abonnement par forte transition $^{74}$ (puisque selon le mode de l'acquisition par attribution) fait venir l'information à l'usager, l'actualité interne de la production universitaire est tournée vers l'extérieur (principe traditionnel de l'entreprise). Ce mouvement vers l'extérieur de l'actualité universitaire ouvre l'université sur l'espace public, par ces temps de $"$ tensions importantes $»^{75}$ pour la communication interne. Quant au mouvement vers l'intérieur, inhérent à la communauté universitaire, il se désigne comme non clos sur lui-même. Cette stratégie met sur le devant de la scène l'organisation interactive par la médiatisation d'événements qui activent le lien professionnel comme lien territorial (l'université dans la ville par l'insertion de la ville dans l'université); le feed-back (caractéristique de l'HI) optimisant le lien professionnel interne.

Toutefois, qui écoutera la web radio d'une université s'il ne fait pas partie de l'université ? À ce sujet nous souhaitons simplement remarquer que les répercussions sémiotiques de l'HI pour la $\mathrm{CdO}$ universitaire portent sur la puissance d'une innovation structurelle, par la complémentarité entre communications verticale et transversale. Si avec l'ENT la relation de base de présupposition réciproque entre communication externe et interne devient complémentaire (l'information concentrique tend vers l'excentrique), avec la web radio nous constatons un autre niveau de déplacement de valeur, celui de la communication transversale qui entre en relation de contrariété (ou de présupposition réciproque) avec la communication verticale.

Reste à identifier la place de la communication horizontale dans ce carré sémiotique de la catégorie organisation interactive (auquel la présente étude permet d'aboutir en mettant en perspective). Car l'organisation interactive et ses dispositifs est moins gage de modernité que trait pertinent de communication au service de l'axiologie de l'entreprise universitaire. ENT et web radio sont des innovations en tant que concepts (en termes de marketing, tel le concept du développement durable par exemple). Construits et pensés pour servir le plan de communication et la gestion de l'information, ENT et web radio sont autant de dispositifs de veille pour l'audit à la communication interne où verticalité, mutualité et horizontalité de l'information sont capitales. L'organisation interactive s'érige alors en univers de références pour la $\mathrm{CdO}$. En tant que tel elle mobilise ENT et web radio (malgré leurs places spécifiques dans le dispositif global) comme outils de la communication externe.

74. Dispositif PUSH : procédé de fourniture de l'information via l'Internet, sans que l'internaute ne doive la chercher lui-même. Dicodunet.com, consulté le 7 juin 2011.

75. Andréa Catellani, «La communication interne à l'épreuve du développement durable : analyse de cas en Belgique ", Actes du 17ème colloque de la SFIC, Au coeur et aux lisières des SIC, Dijon, 2010, p. 322. 


\section{BIBLIOGRAPHIE}

ALMEIDA (D') N., LIBAERT Th., La communication interne des entreprises, Paris, Dunod, 2007, 128 p.

AMADO G., GUITTET A., Dynamique des communications dans les groupes, Paris, Armand Colin, 2009, 205 p.

BALMISSE G., La recherche d'information en entreprise, Paris, Hermès Lavoisier, 2007, 277 p.

BERGSON H., La pensée et le mouvant : essais et conférences, Paris, F. Alcan, 1934, 324 p.

BOUDON J., Introduction aux médias, Paris, Montchrestien, 2009, 158 p.

CASILLI A., Les liaisons numériques - vers une nouvelle sociabilité?, Paris, Seuil, 2010, 332 pages.

CATELLANI A., " La communication interne à l'épreuve du développement durable : analyse de cas en Belgique ", Actes du 17ème colloque de la SFIC, Au coeur et aux lisières des SIC, Dijon, 2010, pp. 322-327.

CERTEAU (de) M., Arts de faire, Paris, Gallimard Folio essais, 1990, 374 p.

ECO U., La structure absente, Introduction à la recherche sémiotique, Paris, Mercure de France, 1972, 447 p.

FABRI P., Le tournant sémiotique, Paris, Hermès Lavoisier, 2008, 164 p.

FONTANILLE J., " Du support matériel au support formel » dans ARABYAN M., KLOCK-FONTANILLE I. éd., L'écriture entre support et surface, Paris, L'Harmattan, 2005, pp. 183-200.

FONTANILLE J., "Les métiers de la sémiotique - signification, conception, innovation ", dans Métiers de la sémiotique, FONTANILLE J., BARRIER G. éd., Limoges, Pulim, 1999, pp. 15-26.

FONTANILLE J., Le savoir partagé : sémiotique et théorie de la connaissance chez Marcel Proust, Paris, Hadès-Benjamin, 1987, 227 p.

FONTANILLE J., BARRIER G., " Les métiers de la sémiotique - signification, conception, innovation ", dans Métiers de la sémiotique, Limoges, Pulim, 1999, 183 p.

FONTANILLE J., « Sémiotique du discours », Pulim, Limoges, 1998, 291 p.

FOURRIER Ch., MARTIN-JUCHAT F., LÉPINE V., «Y-a-t-il un modèle de communication dominant dans les organisations ou une hybridation dans les pratiques des communicants ? Retour réflexif du terrain ", Actes du 17ème colloque de la SFIC, $A u$ coeur et aux lisières des SIC, Dijon, 2010, pp. 437- 443.

GANY D., Nouveaux médias : mode d'emploi, Liège, Edipro, 2009, 144 p.

GREIMAS A., COURTÈS J., Dictionnaire raisonné de la théorie du langage, Hachette Université, 1979, 424 p.

JEANNERET Y., préface, Paolo FABRI Le tournant sémiotique, Paris, Hermès Lavoisier, 2008, pp. 3-23.

JEANNERET Y., Y-a-t-il (vraiment) des technologies de l'information? Villeneuve d'Ascq, Presses universitaires du Septentrion, 2011, 198 p. 
KANDINSKY, Du spirituel dans l'art et dans la peinture en particulier, Paris, Denoël Médiations, 1969, 183 p.

KLINKENBERG J.- M., Précis de sémiotique générale, Bruxelles, De Boeck, 1996, 389 p.

LEFEBVRE A., Les réseaux sociaux: de Facebook aux nouveaux intranets, la généralisation des réseaux sociaux, Paris, M21, 2008, 200 p.

MARIN L., «Une mise en signification de l'espace social : manifestation, cortège, défilé, procession (notes sémiotiques) ", Revue Sociologie du sud-est, Sémiotique et Mass-média, n 37-38, CNRS Université de Nice, Publications Université de Provence », 1983.

MILON A., JOUVE M., Communication et organisation des entreprises : approche critique et cas pratiques, Rosny, Bréal, 1996, 287 p.

MITROPOULOU E., "De la pratique médiatique comme topographie d'une mémoire collective ", dans PIGNIER N. et LAVIGNE M. éd., Revue MEI, Paris, L'Harmattan, pp. 87-97.

MITROPOULOU E., Nouveaux Actes Sémiotiques [En ligne], " Média, multimédia et interactivité : jeux de rôles et enjeux sémiotiques », Nouveaux Actes Sémiotiques, 2007, http://revues.unilim.fr/nas/.

MITROPOULOU E., Nouveaux Actes Sémiotiques [En ligne], «Vers une sémiotique du medium : une sémiotique à légitimer ? ", 2008, http://revues.unilim.fr/nas/.

MitROPOULOU E., NOVELLO PAGLIANTI N., Association Italienne de Sémiotique [En ligne], « La sémiotique des médias et ses objets », 2011, http://www. ec-aiss.it/.

OLLIVIER B., Les sciences de la communication : théories et acquis, Paris, Armand Colin, 2007, 284 p.

PIGNIER N. éd., De l'expérience multimédia - usages et pratiques culturelles, Paris, Hermès Lavoisier, 2009, 212 p.

STOCKINGER P., Sémiotique, Communication et Nouvelles technologies de l'Information [En ligne], 2003, www.semionet.fr

Résumé : Les médias occupent des espaces de notre vie professionnelle, aussi. La dimension communicationnelle (vers l'extérieur comme à l'intérieur) est indissociable du dispositif professionnel global. Est-ce plus valable actuellement qu'à d'autres époques ? Peu importe car notre regard n'est pas diachronique. Pour nous, la communication des organisations est le monde de signification du lien professionnel et un objet d'étude pour le sémioticien de la communication. Engageant un plan et des stratégies de communication, elle signifie une série d'actions, des scènes de transformations, des dispositifs au titre d'innovations (effectives ou prétendues) et de cadres de manipulation pour l'information. Nous nous limitons à l'esquisse d'une problématique sémiotique du lien professionnel interactif, caractéristique des technologies contemporaines de la communication autant interne qu'externe des groupes de travail.

Mots-clefs : intranet, lien professionnel, innovation, hyper-information, sémiotique de la communication. 
Abstract : Interactive Organizations: what's innovative in the field of communication? Our professional life too has to deal with media within its framework. The communicative aspect (towards outside as well as inside) cannot be separated from the global professional system. Is this fact more valid nowadays than in the past? It doesn't matter, since our glance about it is not diachronic. From our point of view, communication within organizations establish a world of meanings related to the professional links, and constitute an object of study for the semioticians of communication. Involving a project and strategies of communication, it means series of actions, scenes of processing, devices agreeing with (effective or claimed) innovation, and frames defining the data processing. We shall limit our comment to sketch the problem of semiotic order concerning the professional link considered through its interactive aspect, which is a characteristic of our contemporary technologies of communication, as much as working groups might be internal or external.

Keywords : intranet, professional links, innovation, hyper-information, semiotics of communication. 